\title{
Human Herpesvirus 8
}

National Cancer Institute

\section{Source}

National Cancer Institute. Human Herpesvirus 8. NCI Thesaurus. Code C14327.

A gammaherpesvirus that contributes to the development of Kaposi sarcoma. 